Revue internationale P.M.E.

Économie et gestion de la petite et moyenne entreprise

\title{
La croissance et la décroissance des entreprises aux Pays-Bas
}

\section{Ron Kemp, Wim Verhoeven et Marcel Kreijen}

Volume 14, numéro 3-4, 2001

URI : https://id.erudit.org/iderudit/1008702ar

DOI : https://doi.org/10.7202/1008702ar

Aller au sommaire du numéro

\section{Éditeur(s)}

Presses de l’Université du Québec

ISSN

0776-5436 (imprimé)

1918-9699 (numérique)

Découvrir la revue

Citer cette note

Kemp, R., Verhoeven, W. \& Kreijen, M. (2001). La croissance et la décroissance des entreprises aux Pays-Bas. Revue internationale P.M.E., 14(3-4), 189-210. https://doi.org/10.7202/1008702ar

\section{Résumé de l'article}

Nous avons étudié la dynamique de la vie économique aux Pays-Bas et, en particulier, l'importance des entreprises en croissance rapide. Celles-ci stimulent l'innovation, défient les concurrents et créent de nombreux emplois. Dans la période 1989-1997, près de la moitié des créations brutes d'emplois par les entreprises existantes (711 000) est due à des entreprises en croissance rapide, qui sont pourtant en nombre relativement limité (6\%) par rapport aux entreprises en croissance normale ( $25 \%$ ). Les entreprises en croissance rapide semblent revenir bien souvent à un rythme de croissance inférieur (67 \%), tandis que près de $8 \%$ des entreprises en croissance normale accélèrent leur croissance. Les jeunes entreprises contribuent à augmenter le nombre des entreprises en croissance rapide. Beaucoup d'entreprises croissent par étapes, car elles se heurtent à des plafonnements. Lorsqu'elles ont résolu leurs difficultés, elles peuvent croître à nouveau. Sur ce point, le gouvernement a une mission à accomplir. Les responsables politiques néerlandais s'efforcent de créer des conditions pour favoriser une concurrence dynamique et prévenir les alliances qui entravent le jeu de la concurrence. En outre, ils cherchent à aplanir les obstacles auxquels se heurtent les entrepreneurs, par exemple les faiblesses du marché, et à stimuler la création d'entreprises. Le gouvernement a pris quelques mesures additionnelles en faveur des entreprises en croissance rapide : il a étendu la portée des réseaux, favorisé la formation pour permettre aux entrepreneurs d'acquérir les habiletés nécessaires et, enfin, il a amélioré l'information fournie.
Ce document est protégé par la loi sur le droit d'auteur. L'utilisation des services d'Érudit (y compris la reproduction) est assujettie à sa politique d'utilisation que vous pouvez consulter en ligne.

https://apropos.erudit.org/fr/usagers/politique-dutilisation/ 


\title{
Notes de recherche \\ La croissance et la décroissance des entreprises aux Pays-Bas
}

\author{
Ron KEMP \\ Institut européen sur les PME (EIM) de Zoetermeer et Université de Wageningen \\ Wim VERHOEVEN \\ Institut européen sur les PME (EIM) de Zoetermeer \\ Marcel KREIJEN \\ Ministère des Affaires économiques néerlandais
}

\section{MOTS CLÉS}

\section{Pays-Bas - Période 1989-1997 - Dynamique de la vie économique Entreprises en croissance rapide - Plafonnements temporaires Implications politiques}

\begin{abstract}
LES AUTEURS
RON KEMP est chercheur principal à l'EIM. Ses travaux portent principalement sur l'innovation, le fonctionnement du marché et le développement de l'entreprise. Il est également attaché à temps partiel au Département d'économie d'entreprise de l'Université de Wageningen. II a publié des articles notamment dans Industrial Marketing Management, Research in Marketing, Tijdschrift voor Bedrijfsadministratie et dans divers actes de congrès. Courriel : <rke@eim.nl>.

MARCEL KREIJEN est conseiller auprès du pôle Entrepreneuriat innovateur et jeunes entrepreneurs du ministère des Affaires économiques néerlandaises. Ses activités se rapportent aux domaines suivants: les entreprises en croissance rapide, les entreprises utilisant les technologies de pointe, l'entrepreneuriat et l'enseignement.

WIM VERHOEVEN est principal gestionnaire de comptes à l'EIM. II a participé à de nombreuses études relatives à la problématique de la croissance, à la dynamique des entreprises (création et disparition d'entreprises), aux systèmes de référence pour les PME (à l'échelle nationale et internationale) et à la productivité dans le secteur quaternaire et dans la fonction publique. Par ailleurs, il travaille au développement de modèles permettant de faire des prévisions sur l'évolution des produits, des coûts, des bénéfices, de l'emploi et du financement des entreprises. Courriel : <wve@eim.nl>.
\end{abstract}




\title{
RÉSUMÉ
}

Nous avons étudié la dynamique de la vie économique aux Pays-Bas et, en particulier, l'importance des entreprises en croissance rapide. Celles-ci stimulent l'innovation, défient les concurrents et créent de nombreux emplois. Dans la période 1989-1997, près de la moitié des créations brutes d'emplois par les entreprises existantes (711 000) est due à des entreprises en croissance rapide, qui sont pourtant en nombre relativement limité (6\%) par rapport aux entreprises en croissance normale (25\%). Les entreprises en croissance rapide semblent revenir bien souvent à un rythme de croissance inférieur (67\%), tandis que près de $8 \%$ des entreprises en croissance normale accélèrent leur croissance. Les jeunes entreprises contribuent à augmenter le nombre des entreprises en croissance rapide. Beaucoup d'entreprises croissent par étapes, car elles se heurtent à des plafonnements. Lorsqu'elles ont résolu leurs difficultés, elles peuvent croître à nouveau. Sur ce point, le gouvernement a une mission à accomplir. Les responsables politiques néerlandais s'efforcent de créer des conditions pour favoriser une concurrence dynamique et prévenir les alliances qui entravent le jeu de la concurrence. En outre, ils cherchent à aplanir les obstacles auxquels se heurtent les entrepreneurs, par exemple les faiblesses du marché, et à stimuler la création d'entreprises. Le gouvernement a pris quelques mesures additionnelles en faveur des entreprises en croissance rapide: il a étendu la portée des réseaux, favorisé la formation pour permettre aux entrepreneurs d'acquérir les habiletés nécessaires et, enfin, il a amélioré l'information fournie.

\begin{abstract}
We have studied the dynamics of economic life in the Netherlands and, in particular, the importance of rapidly growing businesses. These companies stimulate innovation, challenge competitors and create a large number of jobs. During the period from 1989 to 1997 , about half of the jobs created by existing companies $(711,000)$ were accounted for by rapidly growing businesses, despite the fact that these are relatively limited in number (6\%) compared with companies growing at a normal pace (25\%). Fast-growing companies seem to revert to a slower growth rate fairly often (67\%), whereas some $8 \%$ of companies growing at a normal pace accelerate their growth rate. New businesses contribute to increasing the number of rapidly growing companies. Many businesses grow in stages, as they hit a ceiling every now and then. Once they have resolved their difficulties, they can start growing again. The government has a role to play in this area. Dutch political leaders are endeavouring to create suitable conditions for dynamic competition and prevent alliances that restrict competition. They are also seeking to eliminate the obstacles that businessmen come up against, for example a weak market, and to encourage new business start-ups. The government has introduced a few additional measures to help fast-growing companies: it has extended the scope of networks, promoted training to give businessmen the skills they need and, lastly, improved the information provided.
\end{abstract}

\section{RESUMEN}

Hemos estudiado la dinámica de la vida económica en los Países Bajos y especialmente la importancia de las empresas en crecimiento rápido, que son importantes para la economía porque estimulan la inovación, fomentan la competencia 
y crean empleo. En el período 1989/1997 aproximadamente la mitad del empleo bruto creado por las empresas existentes (711.000) corresponde a empresas en crecimiento rápido. Éstas constituyen un número relativamente limitado (6\%), comparadas con las de crecimiento normal (25\%). Las empresas en crecimiento rápido al cabo de algún tiempo suelen reducir su ritmo de crecimiento (67\%). Por el contrario, un $8 \%$ de las empresas de crecimiento normal acelera su crecimiento. Las empresas jóvenes aportan una gran contribución al incremento de las empresas en crecimiento rápido. Muchas de ellas crecen en etapas, alcanzando un techo cada vez. Cuando resuelven sus problemas, pueden crecer de nuevo. En este aspecto, el gobierno tiene una misión que cumplir. La política holandesa se esfuerza por crear las condiciones adecuadas para lograr una competencia dinámica y evitar las alianzas que limitan la competencia. Por otra parte, la política trata de eliminar los obstáculos con que tropiezan los empresarios, como las debilidades del mercado, y de estimular la creación de empresas nuevas. El gobierno ha tomado algunas medidas adicionales en beneficio de las empresas en crecimiento rápido: la extensión del alcance de las redes, el fomento de la formación de los empresarios para que adquieran las aptitudes necesarias y la mejora de la información.

\section{ZUSAMMENFASSUNG}

Wir haben die Dynamik des wirtschaftlichen Leben in den Niederlanden studiert und im speziellen die Bedeutung der schnell wachsenden Unternehmen. Diese stimulieren die Innovation, fordern die Konkurrenten heraus und begründen viele Arbeitsplätze. In der Periode von 1989-1997 wurden ungefähr die Hälfte der gesamten Arbeitsplätze (711 000) von schnell wachsenden Unternehmen begründet, die in der Anzahl dennoch relativ limitiert sind (6\%) gegenüber normal wachsenden Unternehmen (25\%).

Die schnell wachsenden Unternehmen scheinen sehr oft auf einen tieferen Wachstumsrythmus zurückkommen. Im Gegensatz dazu beschleunigen einige von normal wachsenden Unternehmen (8\%) ihr Wachstum. Die jungen Unternehmen tragen dazu bei, die Anzahl an stark wachsenden Unternehmen zu erhöhen. Viele Unternehmen wachsen in Etappen und dann stossen sie an Grenzen. Wenn sie ihre Probleme gelöst haben, können die Unternehmen von neuem wachsen.

An diesem Punkt hat die Regierung eine Mission zu erfüllen. Die politischen Verantwortlichen der Niederlande setzen sich zum Ziel, angepasste Rahmenbedingungen zu schaffen für eine dynamische Konkurrenz und die Allianzen vorherzusagen, welche die Konkurrenz einschränkt. Einerseits suchen sie die Hindernisse zu beseitigen, an denen sich die Unternehmer stossen - zum Beispiel die Schwächen des Marktes - und andererseits die Unternehmensgründung zu stimulieren. Die Regierung hat verschiedene zusätzliche Massnahmen getroffen zu gunsten der stark wachsenden Unternehmen: sie hat das Netzwerk ausgedehnt, die Ausbildung zur nötigen Qualität für Unternehmer gefördert und endlich die Informationen verbessert.

Revue internationale P.M.E., vol. 14, $\mathrm{n}^{\text {os }} 3-4,2001$ 


\section{Introduction}

Début 1999, on comptait aux Pays-Bas environ 700000 entreprises privées opérant en dehors du secteur agricole. La population des entreprises est constamment soumise à des changements ; de nouvelles entreprises apparaissent, d'autres croissent, décroissent ou disparaissent. La figure 1 illustre le dynamisme sous forme de graphique.

FIGURE 1

Effet de la dynamique de la vie économique sur l'emploi

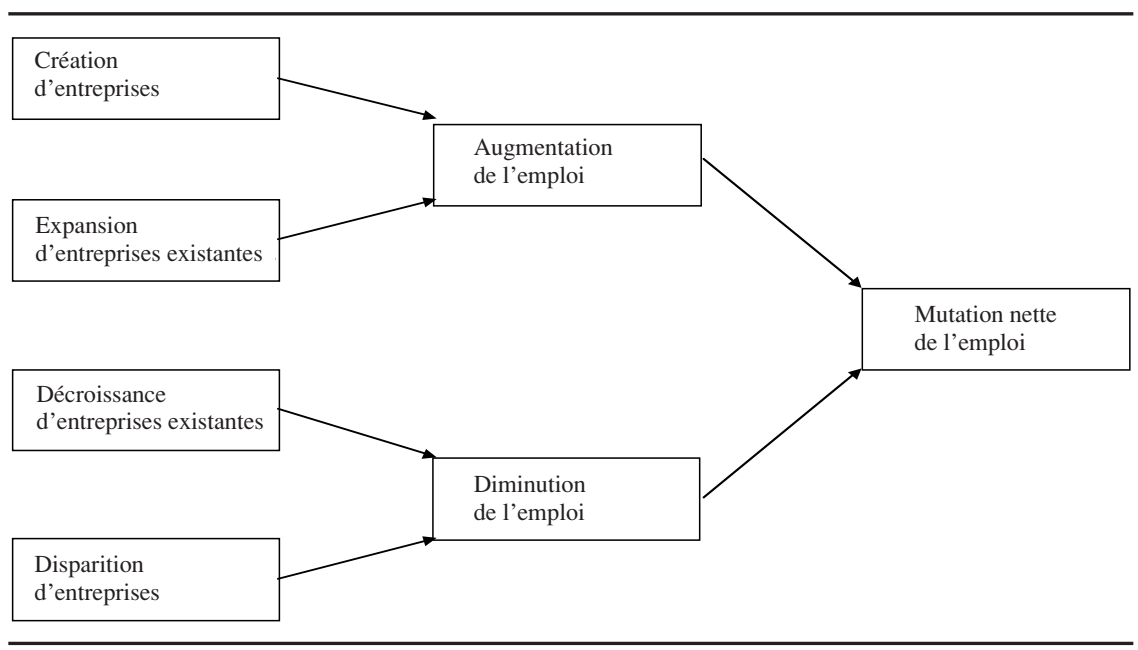

Dans cette étude, nous nous intéressons principalement à la croissance des entreprises existantes. La contribution des nouvelles entreprises à la croissance de l'emploi n'est abordée que de manière indirecte.

L'article est constitué comme suit. Tout d'abord, il traite du concept de la dynamique d'entreprise et de la place qu'y occupent les entreprises en croissance et en décroissance. Ensuite, il étudie brièvement l'effet des nouvelles entreprises et des entreprises ayant cessé leurs activités. Puis, il analyse la croissance et la décroissance des entreprises néerlandaises et le processus de croissance que ces entreprises peuvent suivre. Il termine par la formulation d'un certain nombre d'implications politiques.

\section{Cadre conceptuel}

Combinée au nombre des entreprises de départ, la dynamique de la vie économique est favorable à l'économie, parce qu'elle provoque un comportement innovateur. La dynamique et ses effets sur les innovations augmentent la force de frappe de l'économie, ayant dès lors un effet bénéfique sur la croissance économique. 
Selon un récent rapport de l'OCDE, « la croissance économique dans un pays dépend à long terme de la mise en place et du bon fonctionnement d'un environnement propice à l'innovation et à l'utilisation des nouvelles technologies. Les aides publiques à l'innovation doivent être axées sur les domaines où les effets socioéconomiques sont les plus bénéfiques et permettent de repousser la frontière technologique ${ }^{1} »$. Cette innovation provient souvent d'entreprises relativement jeunes (OCDE, 2000). En particulier les entreprises en croissance rapide affichent un niveau élevé de productivité d'emplois (Van Dijken, Prince et Verhoeven, 1996). Les entreprises en croissance rapide incitent souvent à une plus grande innovation : elles investissent $40 \%$ de plus dans la recherche et développement que les entreprises dont la croissance est plus lente.

Par ailleurs, les entreprises en croissance rapide, de même que les nouvelles entreprises, jouent un rôle très important dans la création d'emplois ; cela a été constaté aux États-Unis (Birch, 1987), mais également aux Pays-Bas. Les entreprises en croissance rapide étant en nombre restreint, on a tendance à sous-estimer leur importance dans l'ensemble de l'économie (Storey, 1997). Dans leur étude sur les entreprises en croissance rapide en Suède, Davidsson et Delmar (2001) nuancent toutefois cette importance. Ils démontrent que la croissance des entreprises existantes est un facteur important dans la croissance de l'emploi ; mais elles perdent aussi de leur impact par suite notamment de restructurations et de hausses de la productivité. Il se peut qu'une partie des entreprises établies depuis longtemps connaisse une croissance rapide tandis que les autres entreprises établies pérennes voient diminuer leur taille. Au total, il semble donc que les entreprises anciennes n'apportent qu'une faible contribution à la croissance de l'emploi. Cette croissance résulte généralement du dynamisme d'entreprises jeunes en croissance rapide. Ces entreprises bénéficient d'un environnement favorable à leur croissance (moyens disponibles, gestion de qualité, possibilités de financement, etc.).

En gros, on peut proposer trois explications à la croissance d'une entreprise. La première relève du modèle des phases de croissance (Garnsey, 2001 ; Welbourne, Meyer et Neck, 1998 ; Eggers, Leahy et Churcill, 1994). Dans ce modèle, la croissance s'explique par la phase dans laquelle se trouve l'entreprise. Le passage d'une phase à l'autre s'accompagne souvent d'une crise. À cet égard, on peut penser à l'envergure du contrôle [span of control] des dirigeants et, au besoin de capital-risque, etc.

L'existence d'une taille optimale pour une entreprise nous fournit une autre explication (Stam, 1999; Trau, 1996). Une entreprise qui n'a pas encore atteint la taille optimale devra croître pour y parvenir si elle veut survivre. La taille optimale est définie en particulier par les investissements risqués, les coûts cachés [sunk costs] et l'importance de la concurrence.

1. Recommandation d'un nouveau rapport de l'OCDE (2001b).

Revue internationale P.M.E., vol. 14, nos 3-4, 2001 
La troisième explication repose sur la supposition que la croissance dépend de la disponibilité de compétences de base et des aptitudes requises (Crijns et Ooghe, 1997; Storey, 1997); celles-ci peuvent porter sur la technologie et le management. Une entreprise qui dispose de compétences de base et de meilleures aptitudes au plan qualitatif peut croître plus vite que les autres entreprises.

\section{Définitions, sources et fiabilité des résultats}

Nous nous attardons ici à l'effet du développement des entreprises existantes sur l'emploi. À cette fin, nous avons classé les entreprises en cinq catégories selon leur croissance. Nous avons choisi d'analyser la croissance sur une période de huit ans pour éviter le piège de la croissance occasionnelle. L'étude porte sur la période 1989-1997, qui est ensuite divisée en sous-périodes 1989-1994 et 1992-1997², ce qui permet de voir si la croissance des entreprises est constante, si elle fléchit ou bien si elle augmente.

La croissance peut se définir de diverses manières. Pour la croissance absolue, on observe la croissance en nombre absolu de salariés ; pour la croissance relative, on considère le pourcentage d'augmentation de l'emploi d'une entreprise. Au regard de la croissance absolue, on constate que ce sont surtout les grandes entreprises qui connaissent une croissance rapide, tandis qu' au regard de la croissance relative ce sont surtout les petites entreprises.

Une croissance de 100 personnes en cinq ans, c'est peu pour une entreprise de 5000 salariés, mais c'est considérable pour une PME. Il est moins difficile pour une entreprise ayant un effectif de deux salariés de doubler le nombre de salariés en cinq ans que pour une entreprise de 200 salariés. C'est pourquoi, pour mesurer la croissance, Birch (1987) a introduit la combinaison de la croissance absolue et de la croissance relative dans ses études. Il applique la formule suivante :

Birch $=\left(\right.$ emploi $_{t}-$ emploi $\left._{t-n}\right) *\left(\right.$ emploi $_{t}-$ emploi $\left._{t-n}\right) /$ emploi $_{t-n}$.

Cependant, même cette méthode entraîne encore un biais en faveur des grandes entreprises. L'indice EIM utilisé est une variante de l'indice Birch. La différence réside dans la contribution de la croissance absolue au seuil de croissance réduit dans l'indice EIM. Cela permet de comparer au mieux, à notre avis, les petites et les grandes entreprises ${ }^{3}$. La croissance sur une période de huit ans est calculée à l'aide de la formule suivante :

2. En faisant chevaucher les périodes, on contrôle les répercussions sur l'économie en 1992 1994.

3. Sans cette réduction, les entreprises de 20000 employés ayant une croissance annuelle de $1 \%$ seraient déjà considérées comme étant en croissance rapide, tandis que les entreprises de deux employés devraient atteindre une croissance de $50 \%$ par an.

Revue internationale P.M.E., vol. 14, $\mathrm{n}^{\text {os }} 3-4,2001$ 
$\mathrm{EIM}=\left(\text { emploi }_{t}-\text { emploi }_{t-8}\right)^{0,25} *\left(\right.$ emploi $_{t}-$ emploi $\left._{t-8}\right) /$ emploi $_{t-8}$

On distingue les types de croissance suivants ${ }^{4}$ :

- entreprises en croissance rapide (indice EIM $\geq 3$ );

- entreprises en croissance normale $(0,05 \leq$ indice EIM $<3)$;

- entreprises stables $(-0,05<$ indice EIM $<0,05)$;

- entreprises en décroissance et croissance : entreprises dont l'emploi est en baisse (indice EIM $\leq-0,05$ ) mais dont le chiffre d'affaires augmente fortement (indice EIM pour chiffre d'affaires $\geq 3$ );

- autres entreprises en décroissance (indice EIM $\leq-0,05$ et indice EIM pour chiffre d'affaires $<3$ ).

Les informations concernant les Pays-Bas ont été empruntées au fichier REACH du cabinet Van Dijk. Dans les fichiers, on retrouve les comptes annuels déposés à la chambre de commerce par les entreprises tenues de le faire, à savoir toutes les personnes morales comptant au moins deux salariés. La base de données contient les entreprises individuelles et les filiales. Les filiales ne sont pas tenues de déposer leurs comptes annuels lorsque la société mère assume l'entière responsabilité des filiales.

La base de données REACH comprend actuellement quelque 125000 entreprises, dont 46000 offrent des données utilisables tant pour 1989 que pour 1997. Plus de 171000 entreprises inscrites en 1997 existaient déjà en 1989. Si bien que, presque $27 \%$ de l'ensemble des entreprises ont été intégrées à l'étude. Les résultats ont fait l'objet d'une extrapolation à l'aide de coefficients, par secteur et par taille des entreprises, pour l'ensemble de la population.

Les deux sections suivantes analysent l'importance et les caractéristiques des cinq types de croissance.

\section{Dynamique de la vie économique}

La dynamique de la vie économique est influencée par la création et la disparition des entreprises et par le développement des entreprises existantes ${ }^{5}$; cela a des conséquences sur l'emploi.

4. Pour la période de huit ans, on a adopté une valeur de 3 pour délimiter les entreprises en croissance rapide; pour la période de cinq ans, on applique une valeur de 1,5. Dans les deux cas, l'augmentation moyenne annuelle de l'emploi est similaire.

5. L'apparition d'une nouvelle entreprise immatriculée au registre du commerce avec une nouvelle activité, fondée par un nouvel entrepreneur ou un entrepreneur existant, dans laquelle l'entrepreneur est actif au moins une heure par semaine et qui génère un chiffre d'affaires.

Revue internationale P.M.E., vol. 14, $\mathrm{n}^{\text {os }} 3-4,2001$ 


\subsection{L'importance des nouvelles entreprises}

Il est ressorti d'une étude précédente (Bangma et Verhoeven, 2000a) que 60000 entreprises ont été créées aux Pays-Bas en 1998 : environ 42000 entreprises individuelles et 18000 nouvelles filiales. Les nouvelles entreprises représentent $10 \%$ de la population des entreprises. Pour expliquer ce nombre, il convient de s'arrêter aux facteurs culturels (Wildeman et al., 1998; Bosma et Zwinkels, 1999). Le nombre des entreprises ayant cessé leurs activités s'approchait de 34000 en 1998. Plus de $25 \%$ des entreprises disparues semblent avoir moins de 1,5 an d'existence (Bosma et Zwinkels, 1999). L'apparition et la disparition assurent conjointement le renouvellement des entreprises existantes.

Les nouvelles entreprises individuelles ont créé ensemble près de 61000 emplois en 1998. Les nouvelles filiales ont à leur tour contribué à la création de 52000 emplois. En 1998, la perte totale d'emplois due à la fermeture d'entreprises s'élève à environ 78000 postes. Cette perte est attribuée en grande partie aux nouvelles entreprises, puisque $47 \%$ des entreprises qui ont cessé leurs activités ont moins de 5,5 ans.

\subsubsection{Croissance et décroissance des entreprises existantes}

La dynamique de la vie économique ne relève pas uniquement de la création de nouvelles entreprises et de la disparition d'entreprises existantes. Cette dynamique et ses conséquences sur l'emploi s'expliquent aussi en grande partie par le fait que les entreprises existantes croissent, restent stables ou décroissent. Aux Pays-Bas, la majorité des entreprises (environ 77 \%) est restée en 1998 à peu près à leur taille de 1997. Cette année-là, $7 \%$ des entreprises ont régressé, ce qui représente une perte de presque 117000 emplois. Les $16 \%$ restants ont assuré la croissance de l'emploi. Cela implique la création d'environ 250000 emplois, représentant près de $70 \%$ de la création totale d'emplois en 1998. Ce sont en particulier les entreprises qui croissent rapidement qui semblent être responsables de la part non proportionnelle de la création d'emplois (Storey, 1997). L'importance pour l'emploi des entreprises en croissance rapide est également reconnue par le ministère des Affaires économiques. Une note intitulée «Snelgroeiende ondernemingen in Nederland» (« Les entreprises en croissance rapide aux Pays-Bas », Ministère des Affaires économiques, 1998) indique qu'au cours de la période 1989-1994, 8 \% des entreprises dont la croissance est la plus rapide sont responsables de plus de la moitié de la création totale brute d'emplois. 


\section{Les différents types de croissance pendant la période 1989-1997}

\subsection{L’importance des différents types de croissance}

\subsubsection{Les entreprises en croissance rapide}

Au cours de la période 1989-1997, environ $6 \%$ des entreprises des Pays-Bas peuvent être considérées comme des entreprises en croissance rapide (voir figure 2). On les retrouve dans presque tous les secteurs de l'économie mais en majorité dans la prestation de services aux entreprises et, en moindre nombre, dans l'industrie manufacturière et dans l'industrie du bâtiment et des travaux publics. L'augmentation moyenne de l'emploi d'une entreprise en croissance rapide se situait dans la période 1992-1997 à environ 12 \% par an. Par ordre de grandeur, on retrouve les entreprises en croissance rapide en majorité dans les plus grandes entreprises. Dans la catégorie des entreprises comptant moins de 10 salariés, $4 \%$ seulement de ces entreprises sont en croissance rapide, contre quelque $21 \%$ dans la catégorie des entreprises de plus de 100 salariés.

FIGURE 2

Répartition des entreprises existantes suivant le type de croissance, 1989-1997 (\%)

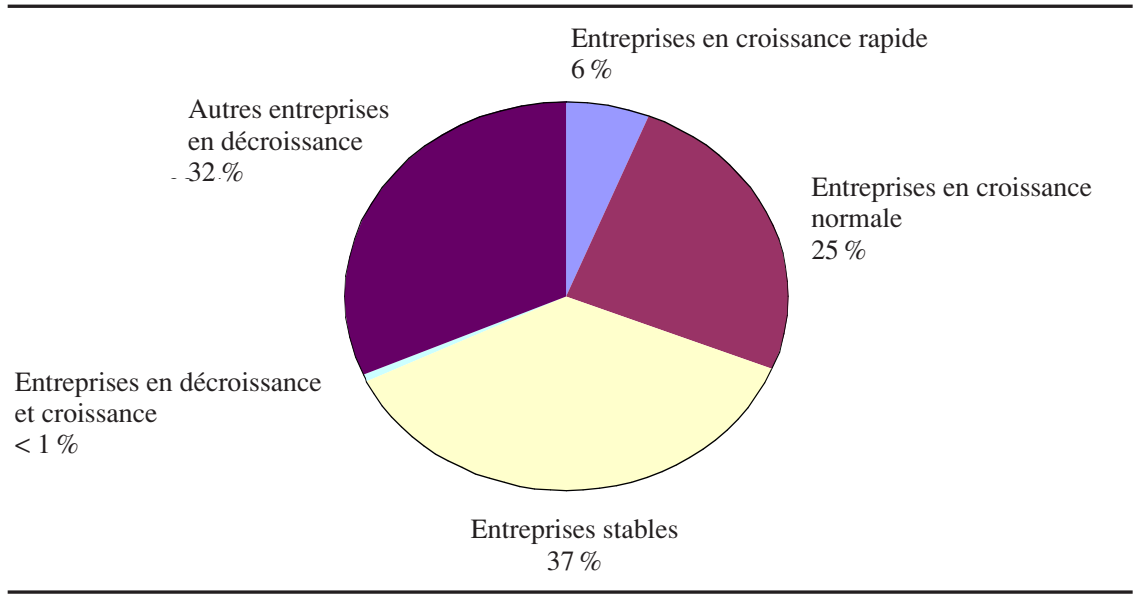

\subsubsection{Les entreprises en croissance normale}

Constituant $25 \%$ des entreprises existantes, les entreprises en croissance normale représentent un groupe important. L'augmentation moyenne annuelle de l'emploi se situait dans la période 1989-1997 aux alentours de 6\%. On retrouve ce type plus

Revue internationale P.M.E., vol. 14, nos 3-4, 2001 
souvent parmi les entreprises comptant de 10 à 100 salariés ou plus de 100 salariés. Quand on regarde par secteur, on les trouve pour plus de la moitié dans le commerce de gros et de détail et dans l'industrie des transports et, pour le reste, dans la restauration et l'hôtellerie et dans les services aux particuliers.

\subsection{Les entreprises stables}

Environ $37 \%$ de toutes les entreprises existantes sont des entreprises stables. La taille des entreprises ne change guère au cours de la période 1989-1997. Il s'agit surtout de petites entreprises employant moins de 10 salariés. On les trouve moins souvent dans la catégorie des entreprises de 10-100 salariés et dans la catégorie des 100 salariés ou plus ${ }^{6}$.

\subsubsection{Les entreprises en décroissance et croissance}

Une catégorie spéciale est constituée par les entreprises dont l'emploi est en décroissance, mais dont le chiffre d'affaires augmente fortement, ce que l'on appelle les entreprises en décroissance et croissance. L'emploi y a diminué de 6,5\% par an avec une croissance moyenne du chiffre d'affaires de $12,5 \%$. Seul un petit nombre d'entreprises peut être ainsi caractérisé pendant cette période de huit ans; on les retrouve en fait parmi les grandes entreprises.

\subsubsection{Les autres entreprises en décroissance}

La dernière catégorie est constituée par les autres entreprises en décroissance. Outre l'emploi en régression, on y observe un chiffre d'affaires modéré ou même négatif ; l'emploi recule chaque année de $6 \%$. Environ $32 \%$ de l'ensemble des entreprises appartiennent à ce groupe. Elles œuvrent le plus souvent dans l'industrie manufacturière, dans la restauration et l'hôtellerie et dans les services aux particuliers. Au plan de la taille, on trouve ces autres entreprises en décroissance en majorité parmi les grandes entreprises.

\subsection{Les caractéristiques des types de croissance}

\subsubsection{Les entreprises en croissance rapide}

Les entreprises en croissance rapide font surtout des affaires en exploitant des créneaux. Leur chiffre d'affaires est élevé et l'assortiment de leur production est une combinaison de produits spécifiques nouveaux et existants. L'exportation n'est pas

6. Aux Pays-Bas, les PME sont définies comme étant des entreprises de moins de 100 salariés.

Revue internationale P.M.E., vol. 14, nºs 3-4, 2001 
indispensable. Elles recherchent les niches sur le marché. Elles se distinguent des autres entreprises par un niveau élevé de la productivité du travail et une stratégie orientée vers l'innovation. En moyenne, elles investissent $40 \%$ de plus dans le développement de nouveaux produits que les entreprises qui ont une croissance plus faible. Le développement des nouveaux produits est l'un des facteurs déterminants de leur compétitivité. En outre, elles ont une structure organisationnelle plus moderne et une politique visible en matière de gestion des ressources humaines $(\mathrm{RH})$. Elles investissent davantage dans leurs salariés que les entreprises en croissance plus faible. Par exemple, les entreprises en croissance rapide consacrent près de $70 \%$ de plus de temps à la formation des salariés que celles en croissance moindre. Elles collaborent avec d'autres entreprises aussi essentiellement dans un cadre informel (Ministère des Affaires économiques, 1998; Verhoeven, 1997). Ces entreprises accordent beaucoup d'importance à l'extension de la capacité, si bien que le développement de la productivité du travail stagne ou même marque un recul. En général, les entreprises en croissance rapide sont plus jeunes que les entreprises ayant un autre type de croissance.

\subsubsection{Les entreprises en croissance normale}

Les entreprises en croissance normale ont un chiffre d'affaires qui augmente moins rapidement que celui des entreprises en croissance rapide, leur production repose principalement sur des produits standard et leur stratégie est plutôt orientée vers le contrôle des coûts. Cependant, elles réagissent rapidement aux nouvelles situations par de nouveaux produits. Tant le niveau que le développement de la productivité du travail se situent aux alentours de la moyenne nationale.

\subsubsection{Les entreprises stables}

Quantité de ces entreprises, notamment celles qui opèrent à l'échelon local, choisissent délibérément de ne pas croître. Leur stratégie est de se mettre en attente. Le niveau de productivité se situe en dessous de la moyenne, de même que le développement.

\subsubsection{Les entreprises en décroissance et croissance}

Les entreprises en décroissance et croissance procurent un chiffre d'affaires plus important avec un effectif réduit. Ces entreprises bénéficient généralement d'une position forte sur le marché extérieur, mais leur niveau de productivité et leur rentabilité sont relativement bas. Leur stratégie est fortement orientée vers la gestion des coûts ; elles sous-traitent davantage que les autres entreprises. En outre, certaines de ces entreprises sont en restructuration permanente.

Revue internationale P.M.E., vol. 14, nºs 3-4, 2001

(C) 2002 - Presses de l'Université du Québec

Édifice Le Delta I, 2875, boul. Laurier, bureau 450, Sainte-Foy, Québec G1V 2M2 • Tél. : (418) 657-4399 - www.puq.uquebec.ca

Tiré de : Revue internationale P.M.E., vol. 14, nos $3-4$, sous la direction de Pierre-André Julien. 


\subsubsection{Les autres entreprises en décroissance}

Les autres entreprises en décroissance sont des entreprises plus anciennes; elles se trouvent dans la phase de maturité et de régression. Ces entreprises sont spécialisées, mais elles appliquent souvent une stratégie d'attente. Leur production est principalement constituée de produits existants et elles recourent peu à l'innovation.

\subsection{Le développement de l'emploi par type de croissance}

L'emploi des entreprises qui ont survécu pendant toute la période 1989-1997 a enregistré une augmentation nette de 97000 postes. La croissance brute au cours de cette période était de 710000 postes, face à la suppression de 610000 postes. Les entreprises en croissance rapide participaient à raison de $49 \%$ à la création brute d'emplois. Les entreprises à la fois en décroissance et en croissance étaient, malgré leur petit nombre, responsables pour $15 \%$ de la décroissance brute (voir figure 3).

FigURE 3

Effet de la dynamique des entreprises sur l'emploi 1989-1997

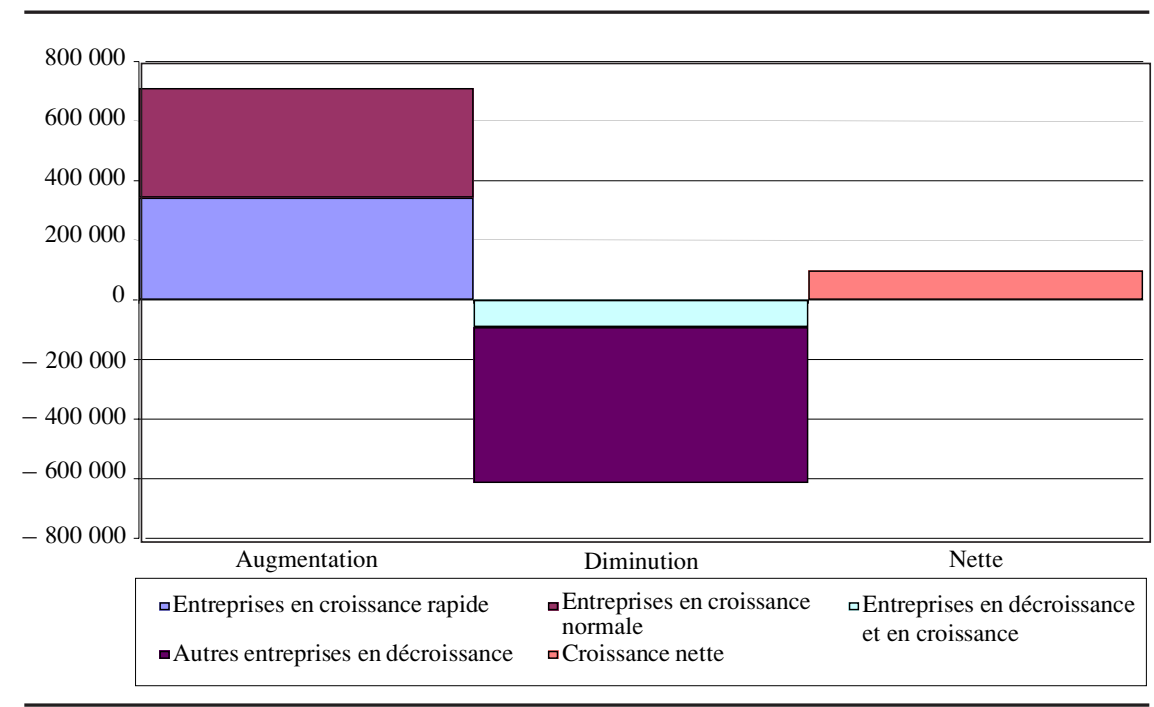

Source : EIM. 


\subsection{Comparaison des deux périodes partielles 1989-1994 et 1992-1997}

Lorsqu'on divise la période 1989-1997 en sous-périodes 1989-1994 et 1992-1997, il est possible d'étudier les changements qui interviennent au plan de la croissance ${ }^{7}$. La figure 4 illustre les schémas de croissance des entreprises pendant les deux périodes. Au cours de la période 1992-1997, on compte moins d'entreprises en croissance rapide que pendant la période 1989-1994; c'est le cas surtout des petites entreprises, en particulier dans le commerce. La proportion d'entreprises stables et d'entreprises en décroissance et en croissance est également moindre dans la période 1992-1997. En revanche, la proportion des entreprises en croissance normale et surtout des entreprises en décroissance est plus élevée en 1992-1997.

FIGURE 4

Comparaison des périodes partielles de 1989-1994 et 1992-1997

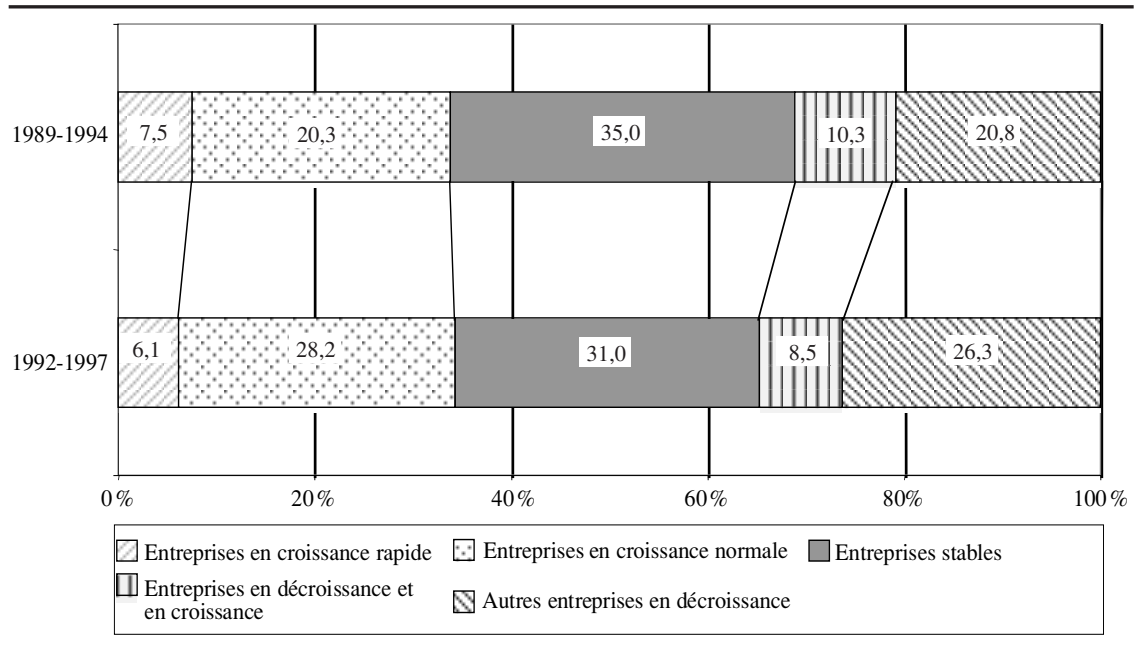

Source : EIM.

Il est étonnant de constater que, pendant les deux sous-périodes, les entreprises en décroissance et en croissance sont clairement représentées : entre $6 \%$ et $9 \%$. Chaque année, l'emploi recule de $5 \%$ alors que le chiffre d'affaires augmente en moyenne de $9 \%$ (1992-1997); on les retrouve en particulier parmi les grosses entreprises. Sans doute ces entreprises suivent-elles une stratégie d'anorexie

7. Pendant les sous-périodes, les entreprises en croissance rapide doivent avoir un indice de croissance EIM supérieur à 1,5. La croissance du chiffre d'affaires doit aussi être supérieure à 1,5 . 
(Witteloostuijn, 1999). La période de huit ans ne compte presque pas d'entreprises en décroissance et en croissance, ce qui indique que la décroissance ne s'observe que pour une période limitée et qu'ensuite on peut à nouveau noter une croissance (l'entreprise passant de la catégorie en décroissance et en croissance à une des catégories stables, en croissance normale ou rapide). Il se peut aussi qu'elles continuent de décroître, mais que l'on ne puisse plus y associer une croissance du chiffre d'affaires (elles tombent alors dans la catégorie des autres entreprises en décroissance).

Pour voir d'où proviennent les entreprises en décroissance et en croissance, il est intéressant de retracer l'origine des entreprises appartenant à un type de croissance déterminé dans la période 1992-1997.

Le tableau 1 montre l'évolution des divers schémas de croissance au cours de la période 1992-1997. Chaque type de croissance semble présenter un noyau fixe d'entreprises appartenant pour les deux périodes au même schéma de croissance, moins souvent des entreprises en décroissance et en croissance et des entreprises en croissance rapide. Seul un cinquième des entreprises en décroissance et en croissance de la période 1992-1997 était aussi en décroissance et en croissance en 19891994. Parmi les entreprises actuellement en croissance rapide (10 100), un tiers (4000) existait déjà dans la période 1989-1994. Ces schémas de croissance semblent donc être les moins stables dans le temps. Pour les autres types de croissance, environ $40 \%$ à $50 \%$ appartenaient au même type de croissance. Parmi les entreprises en croissance rapide en 1992-1997, environ un tiers connaissait auparavant une croissance normale ; ces entreprises ont donc été capables d'accélérer leur croissance. Les entreprises stables et en décroissance franchissent rarement, sinon jamais, le pas vers la croissance rapide. Les entreprises en croissance rapide se retrouvent surtout parmi les nouvelles entreprises. Un cinquième des entreprises en croissance rapide (1992-1997) a été creé dans la période 1989-1991.

Les entreprises en décroissance constituent également un groupe intéressant. Il apparaît que de nombreuses entreprises appartenant à la catégorie des entreprises en décroissance (1989-1994) ont su trouver à nouveau la voie de la croissance. Cette voie passe en particulier par les entreprises en décroissance et en croissance. Même si les entreprises continuent de décroître, elles arrivent à réaliser un chiffre d'affaires raisonnable. Toutefois, près de $40 \%$ des entreprises en décroissance en 1992-1997 appartiennent toujours à ce groupe dans la période 1989-1994. De plus, les entreprises en décroissance se répartissent en entreprises en croissance normale, entreprises stables et entreprises en croissance et en décroissance. Les entreprises en croissance rapide ne passent presque jamais dans la catégorie des entreprises en décroissance. 
TABLEAU 1

Schéma de la modification du rythme de croissance pour les périodes 1989-1994 et 1992-1997

\begin{tabular}{|c|c|c|c|c|c|c|}
\hline & & \multicolumn{5}{|c|}{ Période 1989-1994 $\rightarrow$} \\
\hline & & $\begin{array}{l}\text { Entreprises } \\
\text { en } \\
\text { croissance } \\
\text { rapide }\end{array}$ & $\begin{array}{c}\text { Entreprises } \\
\text { en } \\
\text { croissance } \\
\text { normale }\end{array}$ & $\begin{array}{c}\text { Entreprises } \\
\text { stables }\end{array}$ & $\begin{array}{c}\text { Entreprises } \\
\text { en } \\
\text { décroissance }\end{array}$ & $\begin{array}{c}\text { Nombre } \\
\text { total } \\
\text { d'entreprises } \\
1989-1994\end{array}$ \\
\hline & $\begin{array}{l}\text { Entreprises en } \\
\text { croissance rapide }\end{array}$ & 4000 & 4500 & 1700 & 1900 & 12100 \\
\hline \multirow[t]{4}{*}{$\begin{array}{l}\text { Période } \\
1992-1997\end{array}$} & $\begin{array}{l}\text { Entreprises en } \\
\text { croissance normale }\end{array}$ & le 4400 & 24500 & 12900 & 14300 & 56100 \\
\hline & Entreprises stables & 700 & 10200 & 26200 & 12900 & 50000 \\
\hline & $\begin{array}{l}\text { Entreprises } \\
\text { en décroissance }\end{array}$ & 1000 & 9000 & 11800 & 31200 & 53000 \\
\hline & $\begin{array}{l}\text { Nombre total } \\
\text { d'entreprises } \\
1992-1997\end{array}$ & 10100 & 48200 & 52600 & 60300 & 171200 \\
\hline
\end{tabular}

Source : EIM.

\subsubsection{Les jeunes entreprises}

Le figure 5 donne la répartition des jeunes entreprises suivant le type de croissance. Dans ce contexte, une jeune entreprise est une entreprise comptant deux salariés ou plus, qui a été créée entre 1989 et 1992 et qui existe encore fin 1997. On trouvait approximativement 33000 jeunes entreprises de ce type aux Pays-Bas en 1997. Comparées aux entreprises pérennes, les jeunes entreprises comptent davantage d'entreprises en croissance rapide et moins d'entreprises en décroissance. Il n'est donc pas très étonnant qu'une partie conséquente des entreprises en croissance rapide de la période 1992-1997 soit constituée de jeunes entreprises.

\subsection{Les plafonnements temporaires}

Nous avons vu plus haut que les entreprises suivent une ligne irrégulière de croissance; le rythme de leur croissance s'accélère ou régresse. Il convient de se demander pourquoi.

Comme nous l'avons dit, il est possible d'expliquer de trois manières le changement de rythme de beaucoup d'entreprises en croissance (The Economist, 1999) :

1. Chaque entreprise doit passer à travers des phases de croissance différentes et la transition d'une phase à l'autre s'accompagne d'une crise. À titre

Revue internationale P.M.E., vol. 14, $\mathrm{n}^{\text {os }} 3-4,2001$ 
FiguRE 5

La répartition des jeunes entreprises suivant le type de croissance, 1992-1997 (\%)

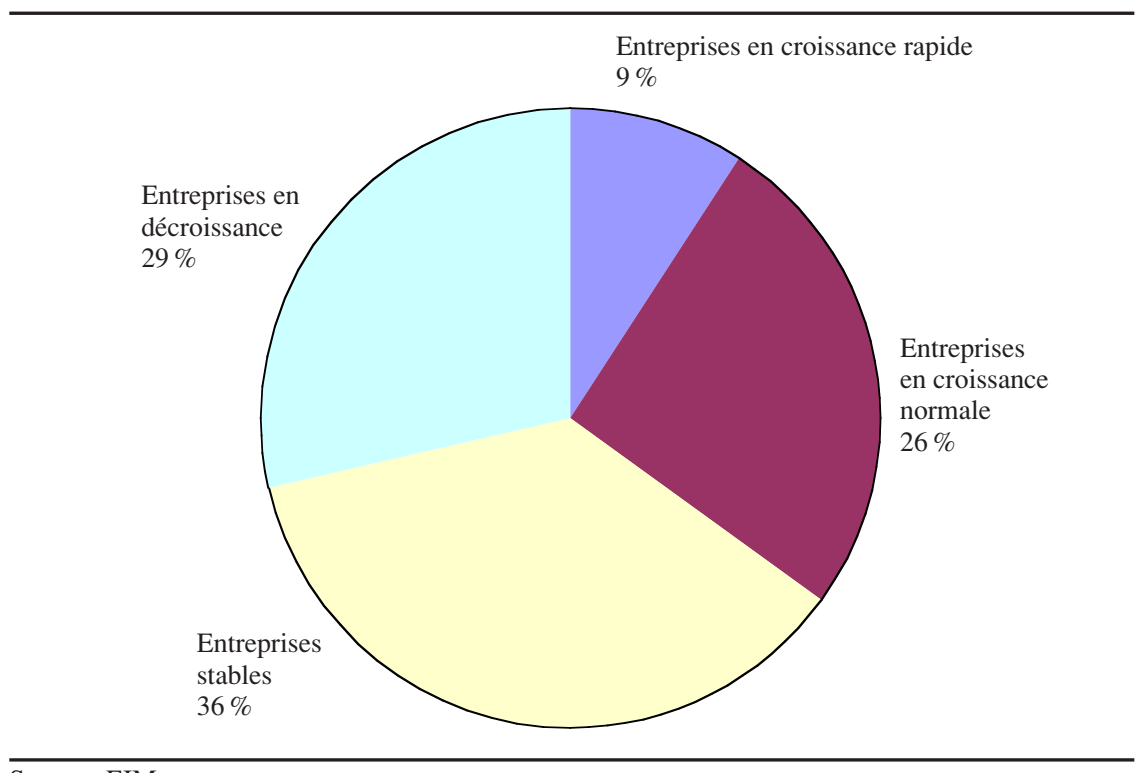

Source : EIM.

d'exemple de crise, nous pouvons mentionner la situation résultant d'une augmentation de l' envergure du contrôle, l'entrepreneur n'étant plus capable de bien gérer son entreprise.

2. Toutes les entreprises en arrivent à une taille optimale. La croissance est une phase temporaire qui permet d'atteindre cette taille optimale.

3. La croissance d'une entreprise dépend des compétences en matière d'organisation.

Chacune de ces explications est valable, mais les hypothèses 1 et 3 posent des problèmes. Nombre d'entreprises en croissance rapide se heurtent aux barrières de croissance, appelées «plafonnements temporaires ». Des obstacles entravent le développement de l'entreprise : la complexité de l'organisation double, la décision de pénétrer le marché extérieur, la formulation d'une stratégie innovatrice, le choix de mettre en œuvre une stratégie personnelle, etc. (voir figure 6).

Généralement, les entreprises en croissance rapide sont capables de dépasser ces plafonnements. Mais certaines entreprises échouent et stagnent à un rythme plus modéré. 
FiguRE 6

\section{Plafonnements temporaires}

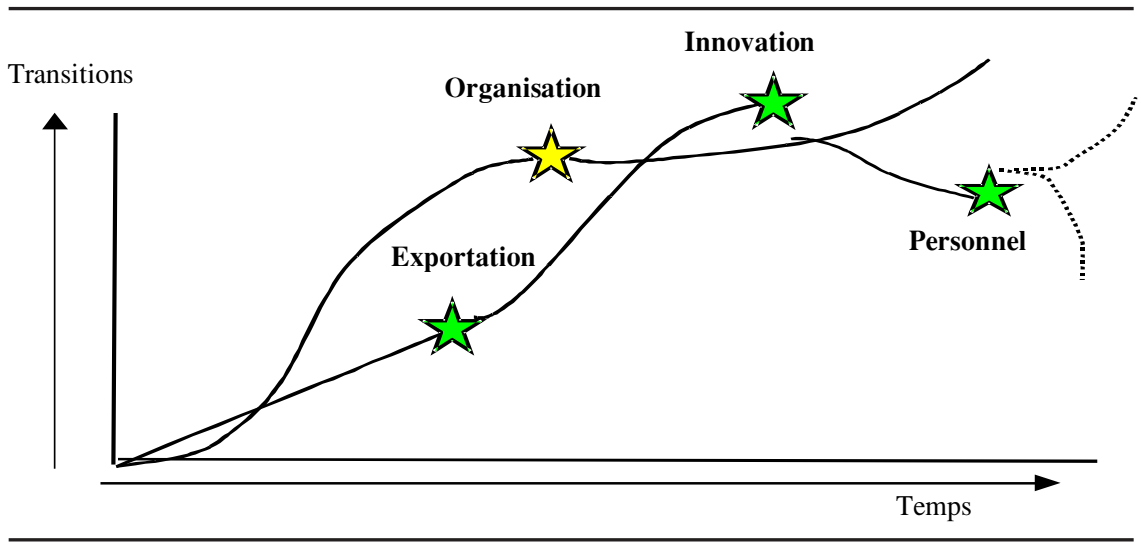

Source : Ministère des Affaires économiques (1999).

Aux Pays-Bas, les entreprises en croissance rapide ont relativement plus de difficultés à briser les plafonnements temporaires que dans d'autres pays. Par exemple, les entreprises en croissance rapide ont besoin de $50 \%$ de plus de temps pour franchir ces obstacles que leurs pendants aux États-Unis (Baljé et Ybema, 1999). Le manque de savoir-faire de l'entrepreneur est l'une des principales causes, mais la faiblesse du conseil et du soutien est également une explication importante. Environ $63 \%$ des entreprises en croissance rapide aux États-Unis font partie intégrante d'un réseau constitué par des entreprises de ce type, contre seulement $27 \%$ aux Pays-Bas (Braaksma, 1999).

\section{Les implications politiques}

Quelles sont les implications pour la politique économique du gouvernement? Comme il est indiqué dans la note de programme (politique) du ministère des Affaires économiques (Ministère des Affaires économiques, 1999), la première mission des responsables politiques est de créer les conditions pour favoriser une concurrence dynamique et prévenir les alliances qui entravent le jeu de la concurrence. En outre, les responsables politiques cherchent à supprimer certains obstacles qui se posent aux entrepreneurs, telles les faiblesses du marché, et à stimuler la création d'entreprises. L'entrepreneuriat est le moteur de l'économie du XXI ${ }^{\mathrm{e}}$ siècle (Baljé et Waasdorp, 2001). L'entrepreneuriat est en effet essentiel pour favoriser le passage d'une croissance reposant sur des facteurs comme ceux que nous avons connus au cours des dernières décennies vers une croissance fondée sur l'innovation pour le siècle à venir (Wijers, 2000). L'apparition et la disparition des entreprises,

Revue internationale P.M.E., vol. 14, nos 3-4, 2001 
que l'on désigne par le terme de brassage [churning], constituent les clés du renouvellement économique. Les entreprises nouvelles et innovantes conçoivent de nouveaux produits, développent de nouveaux marchés et font preuve d'efficacité ; elles incitent ainsi les entreprises existantes à améliorer leur efficacité. À terme, les nouvelles entreprises peuvent devenir des entreprises en croissance rapide. La responsabilité première des hommes politiques est de créer les conditions permettant aux entreprises couronnées de succès de prospérer. La relation entre la politique conduite et les objectifs apparaît à la figure 7. Dans cette figure, trois domaines méritent une attention particulière :

1. Les responsables politiques doivent mettre en place une structure de marché dans laquelle les jeunes entreprises et les entreprises en croissance rapide ont des chances équitables.

2. Les responsables politiques doivent établir des réglementations appropriées et limiter les charges administratives.

3. Les responsables politiques doivent créer un climat productif pour les entreprises dans le domaine des impôts, du financement des entreprises, de la politique régionale et locale (par exemple, elles pourraient attirer l'attention sur les divers guichets qui ne coopèrent pas assez) et de la politique de développement des nouveaux marchés, tant intérieurs qu'extérieurs.

FIGURE 7

Relation entre la politique conduite et les objectifs

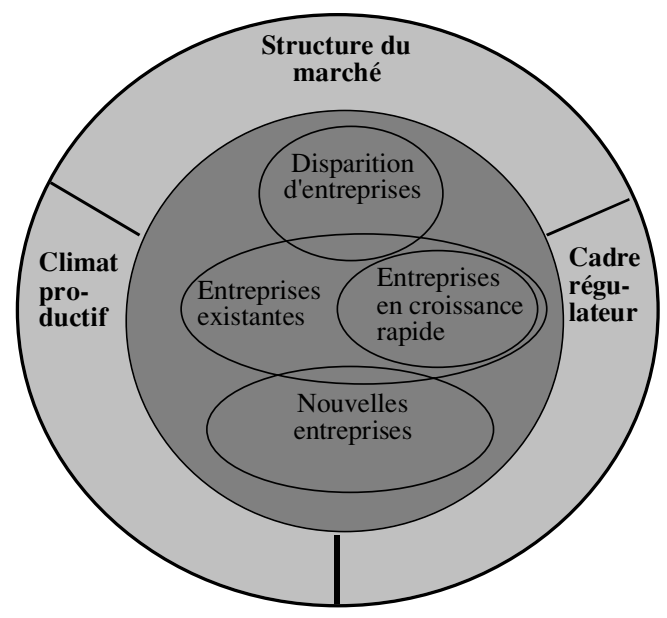

Source : Ministère des Affaires économiques (1999). 
Ces mesures ont un caractère général et sont valables pour toutes les entreprises; d'autres mesures s'appliquent spécifiquement aux entreprises en croissance rapide.

Pour réduire les barrières, les responsables politiques ont instauré quelques mesures supplémentaires concernant les entreprises en croissance rapide. Cette politique a pour but d'éviter que ces entreprises ne retombent dans un rythme plus faible de croissance et, par là même, d'accroître leur nombre. Ces mesures s'articulent autour de trois axes :

1. Améliorer la portée des réseaux et la formation. Le ministère des Affaires économiques a encouragé la création d'une section de l'association paneuropéenne Growth Plus Europe en 2000. Cette association permet d'entrer en contact avec d'autres entreprises en croissance rapide. On peut tirer des enseignements des difficultés éprouvées par des collègues et aider d'autres collègues en suggérant des solutions. Avec le programme « Faites connaissance avec....», le ministère des Affaires économiques propose de jeter un coup d'œil dans les coulisses d'une entreprise en croissance rapide; on y trouve des modèles de rôles qui servent d'outils de comparaison. Le mérite du programme est de favoriser l'échange d'expériences. En outre, un programme éducatif a été développé pour les entreprises en croissance rapide avec la TIAS Business School. Il offre des classes de maîtrise [master classes] pour les entrepreneurs ambitieux. Ces cours s'adressent au groupe d'entrepreneurs situé juste en dessous des 20 entreprises connaissant la croissance la plus rapide (faisant partie du Growth Plus). Elles ont le potentiel pour croître rapidement, mais elles manquent le sommet de peu. De plus, elles sont appelées à résoudre des problèmes similaires à ceux des entreprises en croissance rapide, ce qui les distingue des entreprises «ordinaires ». Le programme aborde des aspects des entreprises en croissance rapide qui sont importants au plan politique.

2. Focaliser l'enseignement sur les qualités indispensables au chef d'entreprise. En accordant plus d'attention à ce sujet pendant la formation, on peut augmenter le nombre des entreprises en croissance rapide. Voilà pourquoi le programme national pour les entrepreneurs a été créé en 2000 avec la collaboration du ministère de l'Éducation, de la Culture et des Sciences. L'idée est de couvrir tous les niveaux de l'éducation, de l'école primaire à l'université. Cette approche néerlandaise de la formation à l'entrepreneuriat a même été qualifiée de bonne pratique dans l'étude internationale des points de référence en matière de politique entrepreneuriale (Stevenson et Lundtsröm, 2001).

3. Améliorer l'information. Les entreprises en croissance rapide peuvent bénéficier de la diffusion des connaissances disponibles sur le phénomène

Revue internationale P.M.E., vol. 14, $\mathrm{n}^{\text {os }} 3-4,2001$ 
des entreprises en croissance rapide et sur les difficultés qu'elles rencontrent. Ces entreprises peuvent apprendre des pratiques exemplaires; un exemple en est donné sur le site Web de US Company Inc (http ://www.inc500.com). Le ministère des Affaires économiques a l'intention de créer un site Web analogue avec Growth Plus Netherlands.

\section{Conclusion}

Dans cet article, nous avons étudié la dynamique des entreprises aux Pays-Bas et, en particulier, l'importance des entreprises en croissance rapide. Dans l'ensemble des entreprises, les entreprises en croissance rapide méritent une attention particulière étant donné qu'elles sont souvent innovantes et qu'elles jouent un rôle important dans la création d'emplois.

Dans la période 1989-1997, quelque $49 \%$ des créations brutes d'emplois par des entreprises sont le fait d'entreprises en croissance rapide. Outre l'importance accordée à ces entreprises, il ressort de cette étude que l'on ne peut certainement pas oublier les entreprises qui croissent normalement. Bien que ces entreprises connaissent une croissance moins rapide, elles sont toutefois à l'origine d'une bonne moitié des emplois créés par les entreprises au cours de la période 1989-1997. En outre, les entreprises en croissance normale peuvent accélérer leur rythme de croissance, tandis que nombre de celles qui ont une croissance rapide ne maintiennent pas longtemps ce rythme de croissance. Près de $40 \%$ des entreprises suivant une croissance normale pendant la période 1992-1997 ont réussi à accélérer leur rythme de croissance pendant la période 1989-1994. Les entreprises en croissance normale sont donc la principale source d'entreprises en croissance rapide. Par ailleurs, un tiers des entreprises en croissance rapide de la période 1989-1994 retrouve un schéma de croissance normal. Cela peut s'expliquer par le fait que les entreprises croissent par étapes, mais aussi par le fait qu'elles se heurtent à des «plafonnements temporaires » et qu'elles doivent dépasser ces plafonnements pour poursuivre leur croissance. Il est donc souhaitable d'accorder une attention particulière aux facteurs favorables à l'accélération de la croissance des entreprises en croissance normale.

Les Pays-Bas sont sur la bonne voie. Ils s'efforcent de supprimer toutes sortes de barrières et mènent depuis peu une politique visant à stimuler les entreprises en croissance rapide. Cette politique est principalement axée sur l'acquisition de connaissances par l'échange d'expériences, la formation et la mise à disposition d'informations. Comme nous l'avons indiqué plus haut, il est primordial d'accorder une attention particulière aux nouvelles entreprises, car, il ne faut pas l'oublier, elles constituent une source importante d'entreprises en croissance rapide. 


\section{Bibliographie}

BALJÉ, S.H. et P.M. WAASDORP (2001), Entrepreneurship in the 21 st Century. The Role of Public Policy, La Haye.

BALJÉ, S.H. et G. YBEMA (1999), « Snel groeien in de informatie-economie (Crô̂tre rapidement dans l'économie de l'information) », Economisch Statistische Berichten, vol. 84, no 4232, p. 905-909.

BANGMA, K.L. et W.H.J. VERHOEVEN (2000a), Het belang van bedrijfstypen voor de werkgelegenheidsontwikkelingen (L'importance des types d'entreprises pour le développement de l'emploi), édition 1999, EIM, Zoetermeer.

BAngma, K.L. et W.H.J. VerhoEVEN (2000b), Groeipatronen van bedrijven, EIM, Zoetermeer.

BIRCH, D.L. (1987), Job Creation in America : How Our Smallest Companies Put the Most People at Work, New York, The Free Press.

Bosma, N.S. et W.S. ZWINKELS (1999), Determinanten van toe- en uittreding in Nederland (Les déterminants de la création et de la fermeture des entreprises aux Pays-Bas), EIM, Zoetermeer.

BRAAKSMA, R. (1999), Snelle groeiers in Nederland en de VS (Les entreprises en croissance rapide aux Pays-Bas et aux États-Unis), EIM, Zoetermeer.

CRIJNS, H. et H. OOGHE (1997), Groeimanagement : Lessen van dynamische ondernemers, Tielt, Lannoo.

DAVIDSSON, P. et F. DELMAR (2001), « Entreprises à forte croissance et leur contribution à l'emploi : le cas de la Suède 1987-1996 », Revue internationale PME, vol. 14, $\mathrm{n}^{\text {os }} 3-4$.

EGGERS, J.H., K.T. LEAHY et N.C. CHURCILL (1994), «Stages of small business growth revisited : insights into growth path and needed leadership / management skills in low and high-growth companies », Présentation à la Babson Entrepreneurship Research Conference.

ELFRING, T. et W. HULSINK (2000), Laat duizend nieuwe bedrijven bloeien (Laissez fleurir mille nouvelles entreprises), ESB, $n^{0} 4251$, p. 312-315.

GARNSEY, E. (2001), «Knowledge-based enterprise in a new economy », Ministry of Economic Affairs / EIM, Entrepreneurship in the Netherlands : New Economy : New Entrepreneurs !

GLOBAL ENTREPRENEURSHIP MONITOR (1999), Executive Report.

HOEVEN, W.H.M. VAN DER et W.H.J. VERHOEVEN (1994), «Creatie en teloorgang van arbeidsplaatsen » (La création et la suppression des emplois), OSA-document de travail, La Haye.

MINISTÈRE DES AFFAIRES ÉCONOMIQUES (1998), Snelgroeiende ondernemingen in Nederland (Les entreprises en croissance rapide aux Pays-Bas), La Haye.

MINISTÈRE DES AFFAIRES ÉCONOMIQUES (1999), De ondernemende samenleving : Meer kansen, minder belemmeringen voor ondernemerschap (La société entreprenante : plus de chances, moins d'obstacles), La Haye.

Revue internationale P.M.E., vol. 14, nos 3-4, 2001 
OCDE (2000), High-growth Firms and Employment, Document de travail STI 2000/3.

OCDE (2001a), Firm Dynamics and Productivity Growth: A Review of Micro Evidence from EOCD Countries, Document de travail du service économique, no 297.

OCDE (2001b), La nouvelle économie : mythe ou réalité?

STAM, E. (1999), «The spatial and sectoral pattern of fast-growing young firms in the Netherlands », Présentation au XXXIX congrès de l'ERSA, 1999, Dublin, Irlande.

STEVEnSON, L. et A. LundSTRÖM (2001), Patterns and Trends in Entrepreneurship / SME Policy and Practice in Ten Economies, Volume 3 de Entrepreneurship Policy for the Future Series.

STOREY, D. (1997), Understanding the Small Business Sector, Londres, International Thompson Business Press.

THE ECONOMIST (1999), The Corporate-Growth Puzzle, 17 juillet, p. 90.

Trau, F. (1996), Why do Firms Grow?, Cambridge, ESRC Centre for Business Research.

VAN DiJKen, K., Y. PRINCE et W. VerhoEVEn (1996), Middelgrote bedrijven in Nederland in internationaal perspectief (Les entreprises moyennes aux Pays-Bas dans une perspective internationale), EIM, Zoetermeer.

VAN DIJKEN, K. et H.-J. HEERES (1999), « Afschaffing vestigingswet : terecht ? (Abrogation de la loi sur l'établissement: à tort ou à raison?)», Economisch Statistische Berichten, vol. 84, no 4234, p. 920-923.

Verhoeven, W.H.J. (1997), «Creatie en verlies van banen in Nederland» (Création et perte des emplois aux Pays-Bas), Présentation à la journée du marché de l'emploi néerlandais 1997, La Haye.

Welbourne, T.M., G.D. MEYER et H.M. NeCK (1998), « Getting past the "entrepreneurial growth ceiling": a longitudinal study of IPO firm growth through solution driven strategies », Communication présentée à la Babson Entrepreneurship Research Conference.

WIJERS, G.J. (2000), « De aaibaarheid voorbij : de kenniseconomie vraagt om fundamenteel andere inrichting van de samenleving », Conférence Den Uyl.

Wildeman, R.E., G. Hofstede, N.G. NoOrderhaven, A.R. ThURIK, W.H.J. VerHOEVEN et S.R.M. WENNEKERS (1998), « Culture's role in entrepreneurship : self-employment out of dissatisfaction », Ribespaper 9815.

Witteloostuijn, A.V. (1999), «De anorexiastrategie : over de gevolgen van saneren » (La stratégie d'anorexie : les conséquences de l'assainir), Amsterdam, de Arbeiderspers. 